between the soluble collagens together with the search for other collagen-like precursors in the cells.

The microtomed tissue slices were incubated in a Krebs medium, which included radioactive proline, for $4 \mathrm{hr}$. at $37^{\circ} \mathrm{C}$. under oxygen. After washing, they were ground and the soluble collagens extracted. The results showed that, in all the purified collagen fractions, the radioactivity of the hydroxyproline was comparable with that of proline, showing that it was derived from the radioactive proline in the medium. In addition, the collagen soluble in neutral salt showed a very much more rapid incorporation of radioactivity than acid-soluble collagen, which in turn showed greater activity than the insoluble collagen. The addition of free hydroxyproline to a culture did not lead to a reduction in the ratio of radioactive hydroxyproline to radioactive proline, while the addition of radioactive hydroxyproline to a medium free of radioactive proline showed no significant incorporation into the collagen. Changes in ratio of specific activities for collagen, hydroxyproline and proline with incubation time, in the presence of radioactive proline, suggest that hydroxylation occurs before incorporation into the protein. Further experiments, in which subcellular fractions were isolated from the tissues, showed that collagens soluble in neutral salt are present in certain fractions. It was suggested that there is a continuous series of collagens of decreasing solubility, of which neutral salt-soluble and acid-soluble collagens are two arbitrary fractions.

A lively discussion followed the paper, and a vote of thanks was proposed by Mr. S. Henson (William Oldroyd and Sons, Ltd.) and seconded by Dr. J. E. Eastoe (Royal College of Surgeons).

A number of analytical techniques were included in the demonstrations at the laboratory. In each instance, part or the whole of the determination was carried through for each group of visitors. Mr. A. A. Leach described his modified Neuman and Logan technique for hydroxyproline determination. $\mathrm{Mr}$. W. G. Cobbett and Dr. A. W. Kenchington demonstrated tyrosine estimation by ultra-violet absorption and by a modified colorimetric method. Dr. G. Stainsby showed how the biuret method could be applied to estimating gelatine concentrations, and Mr. J. T. Taylor carried out the Kodak test for the setting time of a gelatine solution. Mr. A. M. Kragh showed the powerful action of gelatine and glue as flocculation and filtration aids, when used in the correct conditions.

Much interest was aroused by the demonstration by Dr. A. Courts of the properties of a form of soluble collagen which can be isolated from collagenous tissues after they have received pretreatments of the type used in gelatine manufacture (for example, a cold alkaline soak). Substantial percentages of the collagen could be solubilized in this way using $0 \cdot 1 M$ citric acid as solvent. The solution was very viscous but, on warming to $43^{\circ} \mathrm{C}$., a rapid conversion to gelatine occurred, and the viscosity fell more than 40 -fold to a normal value for gelatine. It was possible to precipitate the soluble collagen as fibre by the same procedures as applied to acidsoluble collagens from native tissues. It appears clear that bonds formed when the collagen became insoluble have been broken, enabling the original structural units to be dissolved.

Also on show were equipment for fractionation (W. B. Langston), small-scale gelatine extraction (F. W. Wainewright and A. P. Williams), and the use of $X E 97$ resin for the separation of gelatine components by the method of G. E. Russell (Ilford, Ltd.) (A. A. Leach).
A. G. WARD

\title{
NUMERICAL WEATHER FORECASTING
}

T HE methods of numerical prediction by electronic computer of the atmospheric pressure field now being investigated are based on finite difference approximations to the differential coefficients in the equations of motion. It is required to forecast the variation with time of the height $h$ of an isobaric surface, and the differential coefficients of this quantity occur in the equations.

Starting with a set of values of $h$ at the grid points of a map, the finite-difference approximations can be computed from more than one set of combinations of the values. The effects of using different combinations have been examined in the numerical forecasting research unit of the Meteorological Office, and the results are published in a recent paper entitled "Experiments in Numerical Integration", by E. Knighting, D. E. Jones and Mavis K. Hinds (Quart. J. Roy. Meteor. Soc., 84, 91 ; 1958).

The quantities which have to be computed are the Laplacian $\frac{\partial^{2} h}{\partial x^{2}}+\frac{\partial^{2} h}{\partial y^{2}}$ and a Jacobian $\mathrm{J}(h, \zeta)$, where $\zeta$ is the vertical component of the vorticity, which in turn is expressed as a function of the Laplacian.

The grid points are arranged in square sets. One basic set of grid points is that of the eight points at the centre, corners and mid-points of the sides of a square. These are numbered 0 at the centre, 1, 2, 3, 4 at the mid-points of the sides-with the easternmost point of this set 1 , northern 2 and so on counterclockwise - and $5,6,7,8$ at the corners- with the north-east point 5 and the north-west point 6 and so on as before. Then one finite-difference approximation to the Laplacian is :

$$
\left(h_{1}+h_{2}+h_{3}+h_{4}-4 h_{0}\right) / a^{2}
$$

where $h_{i}$ is the value of $h$ at point $i$ and $2 a$ is the length of a side of the square. Another approx. imation is :

$$
\left(h_{5}+h_{6}+h_{7}+h_{8}-4 h_{0}\right) / 2 a^{2}
$$

Similar, more elaborate combinations apply to the Jacobian.

The authors describe the results of using these different combinations on actual meteorological situations, one of which is the great north-westerly gale of January 31,1953 , which led to the North Sea floods of the following night. Considerable differences are found to occur between the maps of the predicted pressure distributions according to the particular combinations employed. These differences occur even in the centre of the large area dealt with, though that 
is the part in which, because of its distance from the edges with their specified boundary conditions, errors are usually smallest. The difference between two combinations was about 40 per cent of the actual change in two cases and 10 per cent in a third.

These are serious differences, and the authors point out that on some occasions the choice of a geometrical method of making the finite difference approximations has a decisive effect on the forecast. The differences are believed to be associated mainly with the Jacobian, and the authors consider the remedy will have to be sought by using more complicated finite-difference approximations for the derivatives. This would, of course, mean greater complexity and time in the computation.

\title{
RIBONUCLEIC ACIDS IN LIVER CYTOPLASM
}

\author{
By Dr. E. REID and BARBARA M. STEVENS \\ Chester Beatty Research Institute, Institute of Cancer Research : Royal Cancer Hospital, London, S.W.3
}

STUDIES in this laboratory on cytoplasmic $\$$ ribonucleic acid, as influenced by induction of liver tumours ${ }^{1}$ or by a change in hormonal status $^{2-4}$, have emphasized the need for closer study of the ribonucleic acid in the microsomal and supernatant fractions obtained from normal liver. Microsomal ribonucleic acid and supernatantfraction ribonucleic acid may differ in function ${ }^{4}{ }^{5}$; they undergo different changes after adrenalectomy ${ }^{2-4}$, and differ in the time of maximum labelling after injection ${ }^{4}$ of orotic acid- $6{ }^{14} \mathrm{C}^{4}$. Labelling data were compatible with, but did not prove, the postulate $^{7,8}$ that microsomal ribonucleic acid is derived from ribonucleic acid in the supernatant fraction. Supernatant-fraction ribonucleic acid might be derived from acid-soluble nucleotides rather than from nuclear ribonucleic acid ${ }^{9,10}$, but no evidence to support this possible relationship emerged from a comparison of the labelling of acid-soluble uridine nucleotides with that of cytoplasmic ribonucleic acid, after injection of labelled orotic acid ${ }^{6}$.

The study of precursor-product relationships in vivo is hampered by the apparent heterogeneity of the ribonucleic acid in microsomal ${ }^{11-14}$ and supernatant $t^{4,8,11,12}$ fractions. Published procedures ${ }^{12-18}$ were not ideal for the purpose of partitioning the ribonucleic acid among several sub-fractions. This purpose has now been achieved by fractionating tissue proteins (ribonucleoproteins ?) under mild conditions as described below, with tissue samples isolated at different times after injection of orotic acid-6 ${ }^{14} \mathrm{C}$; the time course of ribonucleic acid labelling served as a guide in choosing conditions for separation.

Tissue fractions. Liver homogenates, prepared with $0.25 M$ sucrose from fasted rats given labelled orotic acid, were freed from nuclei and mitochondria, and centrifuged for $90 \mathrm{~min}$. at $14,500 \mathrm{~g}$ to give microsomal and supernatant fractions ${ }^{3,4}$. (The "ultracentrifugal pellet' which can be obtained by re-centrifugation at a higher $g$ value, and which would be included in the 'microsomal' fraction in some laboratories, ${ }^{5}$, resembles our 'ultracentrifugal supernatant' fraction rather than our microsomal fraction in its time course of labelling ${ }^{4,8}$ and in its changes after adrenalectomy ${ }^{2-4}$ or in liver tumours ${ }^{1}$.) The fractions were frozen and stored at $-30^{\circ}$; with microsomal fractions the freezing facilitated the subsequent extraction of ribonucleoprotein.

Analytical methods. Solid samples were counted at infinite thickness, and analysed for ribonucleic acid as previously ${ }^{3,4}$. Almost all the original ribonucleic acid was recovered in the sub-fractions ${ }^{13,17}$. For the subsequent isolation of ribonucleic acid mono- nucleotides, digestion was performed with $1.5 \mathrm{~N}$ potassium hydroxide for $42 \mathrm{hr}$. at $37^{\circ}$, to ensure complete conversion of the ribonucleic acid to mononucleotides $\left.{ }^{19}, 2\right)$. The digest was treated with perchloric acid (to 3 per cent) to remove protein, neutralized with potassium hydroxide, centrifuged to remove perchlorate, and applied to a 'Dowex I' (formate) column. Gradient elution chromatography was carried out with formic acid ${ }^{21}$ to give individual nucleotides, with no attempt ${ }^{20}$ to separate $2^{\prime}$ - and 3 '-isomers. The extinction readings $(260 \mathrm{~m} \mu)$ were assumed to be a valid measure of the amounts present, except for cytidylic acid which, being contaminated with protein ${ }^{20}$, was estimated by analysis of the peak for phosphorus. A further difficulty with cytidylic acid is that some deamination, to give uridylic acid, may occur during the digestion $^{20.22}$; however, this deamination was probably slight because of the presence of tissue protein ${ }^{19}$. Values are expressed relative to guanylic acid as being probably least open to errors of estimation, and are quoted as 'apparent' base composition, since there may still be undetected errors in the present methods. The radioactivity of nucleotide solutions was determined at infinite thinness, with aliquots dried on duralumin planchets.

Supernatant fraction. All fractionations were carried out at about $2^{\circ}$. The procedure for the supernatant fraction (Fig. 1) was based on previous experiments ${ }^{4}$. The ribonucleic acid precipitated by calcium ions partially redissolved on treatment with oxalate $^{14}$. Both the residual ribonucleic acid and the re-dissolved portion which remained soluble on adding streptomycin sulphate ${ }^{18}$ seemed, on the grounds of labelling results, to be worthy of closer examination, together with ribonucleic acid precipitated from the calcium-soluble fraction by ammonium sulphate ${ }^{4}$ at 40 per cent of saturation. There appeared to be differences in base composition (Fig. 1), the last-named fraction being deficient in guanine; but attention is directed particularly to the differences in the time course of ribonucleic acid labelling (Fig. 2). The peak of labelling lay at about $12.5 \mathrm{hr}$. for the ammonium sulphate (40 per cent) precipitate, at about $20 \mathrm{hr}$. for the streptomycin supernatant and not earlier than $42 \mathrm{hr}$. for the residual (oxalate. insoluble) ribonucleic acid. (Note logarithmic axes.)

If the ribonucleic acid with a peak at $12 \cdot 5 \mathrm{hr}$. were the precursor of that in other sub-fractions, it should have relatively high labelling up to $12.5 \mathrm{hr} .{ }^{23}$. Here it is necessary to consider the labelling not of ribonucleic acid as a whole but of the uracil moiety, since, as has now been confirmed, the labelling of 